\title{
Extending EFSMs to specify and test timed systems with action durations and timeouts *
}

\author{
Mercedes G. Merayo, Manuel Núñez and Ismael Rodríguez \\ Dept. Sistemas Informáticos y Programación \\ Universidad Complutense de Madrid, 28040 Madrid, Spain \\ e-mail:mgmerayo@fdi.ucm.es, \{mn,isrodrig\}@sip.ucm.es
}

\begin{abstract}
In this paper we introduce a timed extension of the extended finite state machines model. On the one hand, we consider that output actions take time to be performed. This time may depend on several factors such as the value of variables. On the other hand, our formalism allows to specify timeouts. In addition to present our formalism, we develop a testing theory. First, we define ten timed conformance relations and relate them. Second, we introduce a notion of timed test and define how to apply tests to IUTs.
\end{abstract}

\section{Introduction}

Formal testing techniques $[2,9,14,3,5]$ allow to test the correctness of a system with respect to a specification. Formal testing originally targeted the functional behavior of systems, such as determining whether the tested system can, on the one hand, perform certain actions and, on the other hand, does not perform some non-expected ones. In the last years formal testing techniques also deal with nonfunctional properties such as the time that it takes to perform a certain action. In order to test timed systems, more precisely, the timed behavior of a system, we need a suitable language to formally specify these systems. The time consumed during the execution of a system falls into one of the following categories:

(a) The system consumes time while it performs its operations. This time may depend on the values of certain parameters of the system, such as the available resources.

(b) The time passes while the system waits for a reaction from the environment. In particular, the system can change its internal state if an interaction is not received before a certain amount of time.

A language focussing on temporal issues should allow the specifier to define how the system behavior is affected by both kinds of temporal aspects (e.g., a task is performed if executing the previous task took too much time, if the environment does not react for a long time, if the addition of both times exceeds a

\footnotetext{
* Research partially supported by the Spanish MCYT project TIC2003-07848-C02-01, the Junta de Castilla-La Mancha project PAC-03-001, and the Marie Curie project MRTN-CT-2003-505121/TAROT.
} 
given threshold, etc). In this paper we present a formalism, based on extended finite state machines, allowing to take into account the subtle temporal aspects considered before. Even though there exists a myriad of timed extensions of classical frameworks, most of them specialize only in one of the previous variants: Time is either associated with actions or associated with delays/timeouts. Our formalism allows to specify in a natural way both time aspects. While the definition of the new language is not difficult, mixing these temporal requirements strongly complicates the posterior theoretical analysis. In particular, the definition of timed conformance testing relations is more difficult than usually. The theoretical framework is also complicated by two additional features. First, we consider that variables may influence the timing aspects of the specification. Thus, the execution of an action may take different time values if the value of the variables change. Second, we do not impose any restriction on the deterministic behavior of our machines. This implies again that the same sequence of actions may take different time values to be executed.

We also propose a formal testing methodology allowing to systematically test a system with respect to a specification. Regarding functional conformance we have to consider not only that the sequences of inputs/outpus produced by the implementation must be considered in the specification. We have to take into account the possible timeouts. For example, a sequence of inputs/outputs could be accepted only after different timeouts have been triggered. Let us consider the machines depicted in Figure 1. In these diagrams we use the following notation: A transition labelled by ' $i / o, t$ ' denotes that the execution of the output action $o$ takes time $t$ to be performed after the input $i$ is received; a transition with a label $t$ indicates that a timeout will be applied at time $t$. That is, if after $t$ time units no input is received then the timeout is executed. If we consider $M_{1}$ and $M_{2}$ we can observe that $M_{1}$ is not functionally conforming to $M_{2}$. The sequence $i_{1} / o_{2}$ that can be performed by $M_{1}$ is forbidden by $M_{2}$. On the other hand, if we consider the conformance of $M_{2}$ with respect to $M_{1}$ and we only check the possible sequences of inputs/outputs, $M_{2}$ would conform to $M_{1}$ due to the fact that the unique sequence that can be performed by $M_{2}$ is $i_{1} / o_{1}$. However, this sequence is allowed by $M_{1}$ only in the case that the input has been received after three time units. So, under our conformance framework, $M_{2}$ does not conform to $M_{1}$. We can say the same regarding the conformance of $M_{3}$ with respect to $M_{1}$. On the contrary, this is not the case when considering the conformance of $M_{1}$ with respect to $M_{3}$. The sequences performed by $M_{1}$ are accepted by $M_{3}$ at any time. So, $M_{1}$ functionally conforms to $M_{3}$.

Let us note that testing the temporal behavior of a system affected by nondeterminism requires to face some issues that are not considered by other testing approaches. In particular, contrary to usual approaches, providing an incorrectness diagnosis may require to consider the result of all tests in a test suite, because a single test result could be insufficient to claim the incorrectness of the IUT. ${ }^{1}$ For instance, we may require that, among all the times the IUT may

\footnotetext{
${ }^{1}$ If we consider this statement the other way around then the resulting scenario is the usual one: Passing a test does not allow to claim that the IUT is correct.
} 


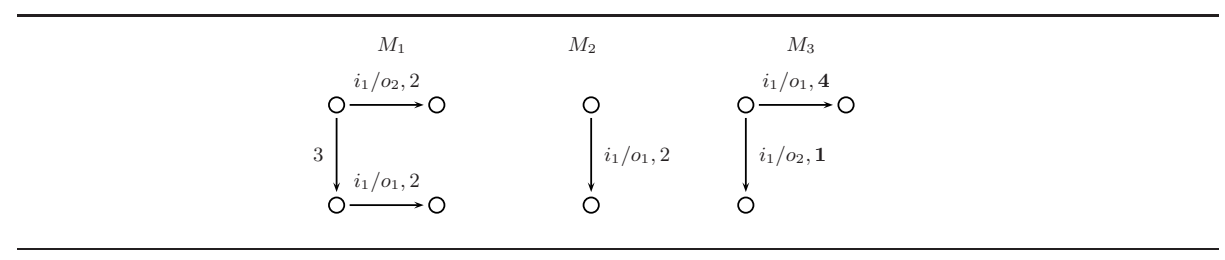

Fig. 1. Examples of functional conformance.

consume to perform a task, one of them is smaller than the corresponding specification time for this task. Hence, if during the application of a test we observe that the IUT takes a long time, then it does not necessarily mean that the IUT cannot do it faster. Regarding temporal performance requirements, our testing methodology will take into account that the system is only responsible for the (a) type consumed time, not for that of (b) type. That is, we have to distinguish between time associated with the performance of tasks and passing of time due to the possible inactivity of the operator of the system.

Our timed conformance relations follow the standard pattern: An implementation is correct with respect to a specification if it does not show any behavior that is forbidden by the specification, where both the functional behavior and the temporal behavior are considered (and, implicitly, how they affect each other). In this paper we present ten different conformance relations. The differences among them come from the effect non-determinism causes in specifications/implementations. We will relate all these notions and propose alternative characterizations for some of them.

In terms of related work, our way to deal with time is completely different to that in timed automata [1]. As we said before, we can associate time with the performance of actions while timeouts can be easily represented. These features do not only improve the modularity of models, but they are also suitable for clearly identifying IUT requirements and responsibilities in a testing methodology. This paper continues the work in [12]. The main advantage with respect to this previous work is that we can now express timeouts, we remove all the restrictions regarding non-determinism of the machines, and we consider more conformance relations. Regarding testing of temporal requirements, there exist several proposals (e.g., $[4,8,15,6]$ ) but most of them are based on timed automata. Moreover, in these approaches tests are independent and the diagnosis of a test does not depend on other tests. By considering that tests are interrelated, we can relate non-determinism and temporal requirements, as well as define and apply several conformance relations where non-determinism is explicitly considered. There are also some time extensions of $\operatorname{FSMs}($ e.g., $[13,7])$ but they do not deal with conformance.

The rest of the paper is structured as follows. In Section 2 we introduce our model. In Section 3 we give our timed conformance relations and provide several examples to show the differences among them. In Section 4 we show how tests are defined and applied to IUTs. 


\section{A timed extension of the EFSM model}

In this section we introduce our notion of timed extended finite state machine. As we have indicated in the introduction of the paper, we will add new features so that the timed behavior of a system can be properly specified. On the one hand, we consider that output actions take time to be executed. These time values will not only depend on the corresponding action to be performed and the state where the machine is placed. Actually, we will also consider that this time value takes into account the current value of the variables. In fact, with this approach we can simulate that the speed with which a task is performed depends on the available resources. On the other hand, we will also consider that the machine can evolve by raising timeouts. Intuitively, if after a given time, depending on the current state, we do not receive any input action then the machine will change its current state.

During the rest of the paper we will use the following notation. A tuple of elements $\left(e_{1}, e_{2} \ldots, e_{n}\right)$ will be denoted by $\bar{e}$. â denotes an interval of elements $\left[a_{1}, a_{2}\right)$. A tuple of intervals is denoted by $\breve{t}$. Let $\breve{q}=\left(\hat{q_{1}}, \ldots, \hat{q_{n}}\right)$ and $\bar{t}=\left(t_{1}, \ldots, t_{n}\right)$. We write $\bar{t} \in \breve{q}$ if for all $1 \leq j \leq n$ we have $t_{j} \in \hat{q}_{j}$. We will use the projection function $\pi_{i}$ such that given a tuple $\bar{t}=\left(t_{1}, \ldots, t_{n}\right)$, for all $1 \leq i \leq n$ we have $\pi_{i}(\bar{t})=t_{i}$. Let $\bar{t}=\left(t_{1}, \ldots, t_{n}\right)$ and $\bar{t}^{\prime}=\left(t_{1}^{\prime}, \ldots, t_{n}^{\prime}\right)$. We write $\bar{t}=\bar{t}^{\prime}$ if for all $1 \leq j \leq n$ we have $t_{j}=t_{j}^{\prime}$. We write $\bar{t} \leq \bar{t}^{\prime}$ if for all $1 \leq j \leq n$ we

have $t_{j} \leq t_{j}^{\prime}$. Finally, we will denote by $\sum \bar{t}$ the sum of all elements of the tuple $\bar{t}$, that is, $\sum_{j=1}^{n} t_{j}$.

Definition 1. Let Time be the domain to define time values, $D_{1}, \ldots, D_{m}$ be sets of values, and let us consider $\mathcal{D}=D_{1} \times D_{2} \times \cdots \times D_{m}$. A Timed Extended Finite State Machine, in the following TEFSM, is a tuple $M=\left(S, I, O, T O, T r, s_{i n}, \bar{y}\right)$ where $S$ is a finite set of states, $I$ is the set of input actions, $O$ is the set of output actions, TO :S $\longrightarrow S \times($ Time $\cup \infty$ ) is the timeout function, $\operatorname{Tr}$ is the set of action transitions, $s_{i n}$ is the initial state, and $\bar{y} \in \mathcal{D}$ is the tuple of initial values of the variables.

An action transition is a tuple $\left(s, s^{\prime}, i, o, Q, Z, C\right)$ where $s, s^{\prime} \in S$ are the initial and final state of the transition, $i \in I$ and $o \in O$ are the input and output action associated with the transition, $Q: \mathcal{D} \longrightarrow$ Bool is a predicate on the set of variables, $Z: \mathcal{D} \longrightarrow \mathcal{D}$ is a transformation over the current variables, and $C: \mathcal{D} \longrightarrow$ Time is the time that the transition needs to be completed.

A configuration in $M$ is a pair $(s, \bar{x})$ where $s \in S$ is the current state and $\bar{x} \in \mathcal{D}$ is the tuple containing the current value of the variables.

We say that $M$ is input-enabled if for all state $s \in S$ and input $i \in I$ there exist $s^{\prime}, o, Q, Z, C$ such that $\left(s, s^{\prime}, i, o, Q, Z, C\right) \in \operatorname{Tr}$.

Given a configuration $(s, \bar{x})$, an action transition $\left(s, s^{\prime}, i, o, Q, Z, C\right)$ denotes that if the input $i$ is received and $Q(\bar{x})$ holds then the output $o$ will be produced after $C(\bar{x})$ units of time, and the configuration will be $\left(s^{\prime}, Z(\bar{x})\right)$. In this paper we consider that time can be discretized, that is, the time domain is isomorphic to $\mathbb{N}$. We will take advantage of this characteristic to simplify some definitions. 
In particular, we will sometimes enumerate the elements of Time simply as $0,1,2$ and so on.

For each state $s \in S$, the application of the timeout function $T O(s)$ returns a pair $\left(s^{\prime}, t\right)$ indicating the time that the machine can remain at the state $s$ waiting for an input action, and the state to which the machine evolves if no input is received on time. We assume that $T O(s)=\left(s^{\prime}, t\right)$ implies $s \neq s^{\prime}$, that is, timeouts always produce a change of the state. We indicate the absence of a timeout in a given state by setting the corresponding time value to $\infty$.

Definition 2. Let $M=\left(S, I, O, T O, T r, s_{i n}, \bar{y}\right)$ be a TEFSM and $c_{0}=\left(s_{0}, \bar{x}_{0}\right)$ be a configuration of $M$. A tuple $\left(s_{0}, s, i / o, \hat{t}, t_{o}, \bar{v}\right)$ is a step of $M$ for the configuration $c_{0}$ if there exist $k \geq 0$ states $s_{1}, \ldots, s_{k} \in S$ such that for all $1 \leq j \leq k$ we have $T O\left(s_{j-1}\right)=\left(s_{j}, t_{j}\right)$ and there exists a transition $\left(s_{k}, s, i, o, Q, Z, C\right) \in T_{r}$ such that $Z\left(\bar{x}_{0}\right)=\bar{v}, \hat{t}=\left[\sum_{j=1}^{k} t_{j}, \sum_{j=1}^{k} t_{j}+\pi_{2}\left(T O\left(s_{k}\right)\right), t_{o}=C\left(\bar{x}_{0}\right)\right.$ and $Q\left(\bar{x}_{0}\right)$ holds. We denote by $\operatorname{Steps}(M, s, \bar{x})$ the set of steps of $M$ for the configuration $(s, \bar{x})$.

We say that $\left(\hat{t}_{1} / i_{1} / t_{o 1} / o_{1}, \ldots, \hat{t}_{r} / i_{r} / t_{o r} / o_{r}\right)$ is a timed evolution of $M$ if there exist $r$ steps of $M\left(s_{i n}, s_{1}, i_{1} / o_{1}, \hat{t}_{1}, t_{o 1}, \bar{y}_{1}\right), \ldots,\left(s_{r-1}, s_{r}, i_{r} / o_{r}, \hat{t}_{r}, t_{o r}, \bar{y}_{r}\right)$ for the configurations $\left(s_{i n}, \bar{y}\right), \ldots,\left(s_{r-1}, \bar{y}_{r-1}\right)$, respectively. We denote by $\operatorname{TEvol}(M)$ the set of timed evolutions of $M$. In addition, we say that $\left(\hat{t}_{1} / i_{1} / o_{1}, \ldots, \hat{t}_{r} / i_{r} / o_{r}\right)$ is a functional evolution of $M$ and we denote by $\operatorname{FEvol}(M)$ the set of functional evolutions of $M$.

Intuitively, a step is an action transition preceded by zero or more timeouts. The interval $\hat{t}$ indicates the time values where the input action could be received. An evolution is a sequence of inputs/outputs corresponding to the action transitions of a chain of steps where the first one begins with the initial configuration of the machine. In addition, timed evolutions include time values which inform us about possible timeouts (indicated by the intervals $\hat{t}_{j}$ ) and the time consumed to execute each output after receiving each input in each step of the evolution.

Example 1. Consider the TEFSM depicted in Figure 2. We suppose that the variables of the TEFSM are given by a tuple $\bar{x} \in \mathbb{R}_{+}^{4}$ and we denote by $x_{i}$ the $i$-th component of $\bar{x}$. Let us assume that the value of the variables is $\bar{x}=(1,2,2,1)$. Next, we give some of the steps that the machine can generate. For example, $\left(s_{1}, s_{2}, i_{1} / o_{1},[0,3), 3,(2,2,2,0)\right)$, represents the transition $t_{12}$ when no timeouts precede it. The input $i_{1}$ can be accepted before 3 units of time pass (this is indicated by the interval $[0,3))$. In addition, $o_{1}$ takes $C_{1}((1,2,2,1))=3$ time units to be performed and the new tuple of variables is $Z_{1}((1,2,2,1))=(2,2,2,0)$. The second one, $\left(s_{1}, s_{4}, i_{1} / o_{2},[3,7), 4,(1,3,1,1)\right)$ is built from the timeout transition associated to the state $s_{1}$ and the action transition $t_{34}$. The step represents that if after 3 units of time no input is received, the timeout transition associated with that state will be triggered and the state will change to $s_{3}$. After this, the machine can accept the input $i_{1}$ before 4 units of time pass, that is, the timeout assigned to the state $s_{3}$. So during the time interval $[3,7)$ if the machine receives an input $i_{1}$ it will emit an output $o_{2}$ and the state will change to $s_{4}$. Similarly, 

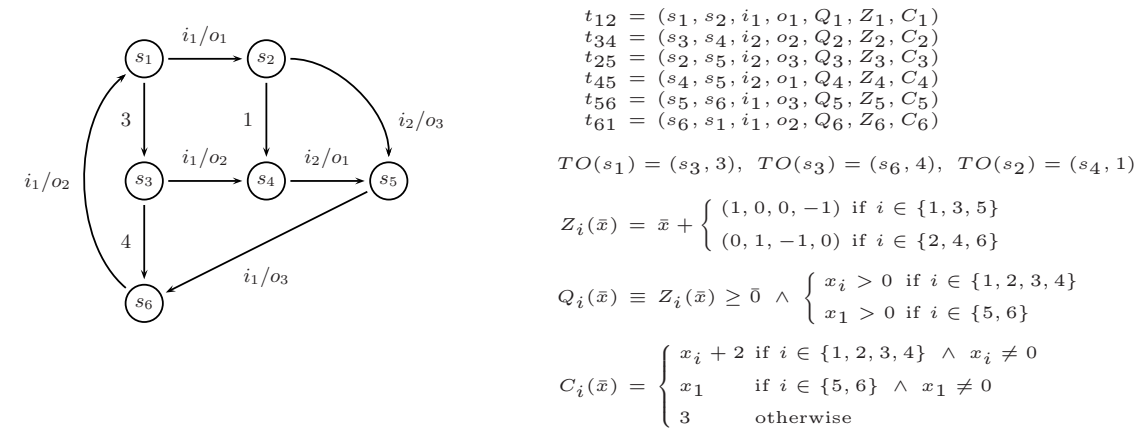

Fig. 2. Example of TEFSMs.

we can obtain the step $\left(s_{1}, s_{1}, i_{1} / o_{2},[7, \infty), 1,(1,3,1,1)\right)$, using the timeout transitions corresponding to $s_{1}$ and $s_{3}$ and the action transition $t_{61}$. All the steps presented, correspond to the configuration $\left(s_{1},(1,2,2,1)\right)$.

Now, we present an example of a temporal evolution built from two steps, and assuming that $s 1$ is the initial state: $\left([7, \infty) / i_{1} / 1 / o_{2},[3,7) / i_{1} / 3 / o_{2}\right)$. The configuration that has been considered for the first step is again $\left(s_{1},(1,2,2,1)\right)$. The configuration that corresponds to the second step is the one obtained after the first step has been performed, that is, $\left(s_{1},(1,3,1,1)\right)$.

Let us note that different instances of the same evolution may appear in a specification as result of the different configurations obtained after traversing the corresponding TEFSM.

In the following definition we introduce the concept of instanced evolution. Intuitively, instanced evolutions are constructed from evolutions by instanciating to a concrete value each timeout, given by an interval, of the evolution.

Definition 3. Let $M=\left(S, I, O, T O, T r, s_{i n}, \bar{y}\right)$ be a TEFSM and let us consider a timed evolution $e=\left(\hat{t}_{1} / i_{1} / t_{o 1} / o_{1}, \ldots, \hat{t}_{r} / i_{r} / t_{o r} / o_{r}\right)$. We say that the tuple $\left(t_{1} / i_{1} / t_{o 1} / o_{1}, \ldots, t_{r} / i_{r} / t_{o r} / o_{r}\right)$ is an instanced timed evolution of $e$ if for all $1 \leq$ $j \leq r$ we have $t_{j} \in \hat{t}_{j}$. In addition, we say that the tuple $\left(t_{1} / i_{1} / o_{1}, \ldots, t_{r} / i_{r} / o_{r}\right)$ is an instanced functional evolution of $e$.

We denote by InsTEvol $(M)$ the set of instanced timed evolutions of $M$ and by InsFEvol $(M)$ the set of instanced functional evolutions.

By abusing the notation, we will sometimes refer to instanced time evolutions such as $\left(t_{1} / i_{1} / t_{o 1} / o_{1}, \ldots, t_{r} / i_{r} / t_{o r} / o_{r}\right)$ as $\left(\bar{t}, \sigma, \bar{t}_{o}\right)$, where $t=\left(t_{1}, \ldots, t_{r}\right), \sigma=$ $\left(i_{1} / o_{1}, \ldots, i_{r} / o_{r}\right)$, and $t_{o}=\left(t_{o 1}, \ldots, t_{o r}\right)$. Similarly, we will also refer to instanced functional evolutions as $(\bar{t}, \sigma)$.

Example 2. As example, if we consider the temporal evolution showed previously, $\left([7, \infty) / i_{1} / 1 / o_{2},[3,7) / i_{1} / 3 / o_{2}\right)$, we have that $\left(8, / i_{1} / 1 / o_{2}, 5 / i_{1} / 3 / o_{2}\right)$ and $\left(12, / i_{1} / 1 / o_{2}, 3 / i_{1} / 3 / o_{2}\right)$ are instanced temporal evolutions. 


\section{3 (Timed) Implementation Relations}

In this section we introduce our implementation relations. All of them follow the same pattern: An implementation $I$ conforms to a specification $S$ if for all possible evolution of $S$ the outputs that the implementation $I$ may perform after a given input are a subset of those for the specification. This pattern is borrowed from $\operatorname{conf}_{n t}$ [10] and it is inspired in ioco [16]. In addition to the non-timed conformance of the implementation, we require some time conditions to hold. For example, we may ask an implementation to be always faster than the time constraints imposed by the specification. Additionaly, we require that the implementation always complies with the timeouts established by the specification.

Next, we formally define the sets of specifications and implementations. A specification is a timed extended finite state machine. Regarding implementations, we consider that they are also given by means of TEFSMs. In this case, we assume, as usual, that all the input actions are always enabled in any state of the implementation. Thus, we can assume that for any input $i$ and any state of the implementation $s$ there always exists a transition $(s, s, i$, null, $Q, Z, C)$ where null is a special (empty) output symbol, the predicate $Q(\bar{x})$ is defined as $\neg \bigvee\left\{Q^{\prime}(\bar{x}) \mid \exists\right.$ a transition $\left.\left(s, s^{\prime}, i, o, Q^{\prime}, Z^{\prime}, C^{\prime}\right)\right\}, Z(\bar{x})=\bar{x}$, and $C(\bar{x})=0$. Let us note that such a transition will be performed when (and only if) no other transition is available for input $i$ (that is, either there are no transitions outgoing from $s$ labelled by $i$ or none of the corresponding predicates hold). Let us note that we do not restrict the machines to be deterministic. Thus, both implementations and specifications may present non-deterministic behavior. This is an important advantage with respect to previous work [12].

First, we introduce the implementation relation $\operatorname{conf}_{f}$, where only functional aspects of the system (i.e., which outputs are allowed/forbidden) are considered while the performance of the system (i.e., how fast are actions executed) is ignored. Let us note that the time spent by a system waiting for the environment to react has the capability of affecting the set of available outputs of the system. This is because this time may trigger a change of the state. So, a relation focusing on functional aspects must explicitly take into account the maximal time the system may stay in each state. This time is given by the timeout of the state.

Definition 4. Let $S, I$ be TEFSMs. We say that $I$ functionally conforms to $S$, denoted by $I \operatorname{conf}_{f} S$, if for each functional evolution $e \in \operatorname{FEvol}(S)$, with $e=\left(\hat{t}_{1} / i_{1} / o_{1}, \ldots, \hat{t}_{r} / i_{r} / o_{r}\right)$ and $r \geq 1$, we have that for all $t_{1} \in \hat{t}_{1}, \ldots, t_{r} \in \hat{t}_{r}$ and $o_{r}^{\prime}, e^{\prime}=\left(t_{1} / i_{1} / o_{1}, \ldots, t_{r} / i_{r} / o_{r}^{\prime}\right) \in \operatorname{InsFEvol}(I)$ implies $e^{\prime} \in \operatorname{InsFEvol}(S)$.

The idea underlying the definition of $\operatorname{conf}_{f}$ is that the implementation does not invent anything for those sequences of inputs that are specified in the specification. Let us note that if the specification has also the property of input-enabled then we may remove the condition "for each functional evolution $e \in \operatorname{FEvol}(S)$, with $e=\left(\hat{t}_{t 1} / i_{1} / o_{1}, \ldots, \hat{t}_{t r} / i_{r} / o_{r}\right)$ and $r \geq 1$ ". Next, we introduce our timed implementation relations. We will distinguish two classes of conformance relations: 
Weak and strong. The family of weak conformance relations demands conditions only over the total time associated to timed evolutions of the implementation with respect to the corresponding timed evolutions of the specification. In contrast, strong conformance relations establish requests over the time values corresponding to the performance of each transition separately. For each of these approaches we define five relations. In the $\operatorname{conf}_{a}^{s}$ and $\operatorname{conf}_{a}^{w}$ relations (conforms always) we consider, for any timed evolution $\sigma$ of the implementation, that if its associated functional evolution $\sigma^{\prime}$ is a functional evolution of the specification then $\sigma$ is also a timed evolution of the specification. In the $\operatorname{conf}_{w}^{s}$ and $\operatorname{conf}_{w}^{w}$ relations (conformance in the worst case) the implementation is forced, for each timed evolution fulfilling the previous conditions, to be faster than the slowest instance of the same evolution in the specification. The $\operatorname{conf}_{b}^{s}$ and $\operatorname{conf}_{b}^{w}$ relations (conforms in the best case) are similar but considering only the fastest instance of the specification. Finally, the relations $\operatorname{conf}_{s w}^{s}$ and $\operatorname{conf}_{s w}^{w}$ (sometimes worst), and $\operatorname{conf}_{s b}^{s}$ and $\operatorname{conf}_{s b}^{w}$ (sometimes best), are similar to the previous relations, but in each case only one instance of each temporal trace of the implementation is required to be as fast as the worst/best instance in the specification.

Definition 5. Let $\bar{t}_{o}=\left(t_{o 1} \ldots t_{o r}\right) \in$ Time $^{r}$. For all instanced functional evolution insfevol $=\left(t_{1} / i_{1} / o_{1}, \ldots, t_{r} / i_{r} / o_{r}\right) \in(\text { Time } \times I \times O)^{r}$, we denote by insfevol $\nabla \bar{t}_{o}$ the instanced timed evolution $\left(t_{1} / i_{1} / t_{o 1} / o_{1}, \ldots, t_{r} / i_{r} / t_{o r} / o_{r}\right)$. Let $S$ and $I$ be TEFSMs. The timed conformance relations are defined as follows:

- (strong always) $I \operatorname{conf}_{a}^{s} S$ iff $I \operatorname{conf}_{f} S$ and for all instanced functional evolution insfevol $\in \operatorname{InsFEvol}(I) \cap \operatorname{InsFEvol}(S)$ we have that $\forall \bar{t}_{i}$

$$
\text { insfevol } \nabla \bar{t}_{i} \in \operatorname{InsTEvol}(I) \Longrightarrow \text { insfevol } \nabla \bar{t}_{i} \in \operatorname{InsTEvol}(S)
$$

- (strong best) $I \operatorname{conf}_{b}^{s} S$ iff $I \operatorname{conf}_{f} S$ and for all instanced functional evolution insfevol $\in \operatorname{InsFEvol}(I) \cap \operatorname{InsFEvol}(S)$ we have that $\forall \bar{t}_{i}$

$$
\text { insfevol } \nabla \bar{t}_{i} \in \operatorname{InsTEvol}(I) \Longrightarrow \forall \bar{t}_{s}:\left(\begin{array}{c}
\text { insfevol } \nabla \bar{t}_{s} \\
\in \operatorname{InsTEvol}(S) \\
\Downarrow \\
\bar{t}_{i} \leq \bar{t}_{s}
\end{array}\right)
$$

- (strong worst) $I \operatorname{conf}_{w}^{s} S$ iff $I \operatorname{conf}_{f} S$ and for all instanced functional evolution insfevol $\in \operatorname{InsFEvol}(I) \cap \operatorname{InsFEvol}(S)$ we have that $\forall \bar{t}_{i}$

$$
\text { insfevol } \nabla \bar{t}_{i} \in \operatorname{InsTEvol}(I) \Longrightarrow \exists \bar{t}_{s}:\left(\begin{array}{c}
\text { insfevol } \nabla \bar{t}_{s} \in \operatorname{InsTEvol}(S) \\
\wedge \\
\bar{t}_{i} \leq \bar{t}_{s}
\end{array}\right)
$$

- (strong sometimes best) $I \operatorname{conf}_{s b}^{s} S$ iff $I \operatorname{conf}_{f} S$ and for all instanced functional evolution insfevol $\in \operatorname{InsFEvol}(I) \cap \operatorname{InsFEvol}(S)$ we have that $\exists \bar{t}_{i}$ such that

$$
\text { insfevol } \nabla \bar{t}_{i} \in \operatorname{InsTEvol}(I) \wedge \forall \bar{t}_{s}:\left(\begin{array}{c}
\text { insfevol } \nabla \bar{t}_{s} \in \operatorname{InsTEvol}(S) \\
\Downarrow \\
\bar{t}_{i} \leq \bar{t}_{s}
\end{array}\right)
$$


- (strong sometimes worst) $I \operatorname{conf}_{s w}^{s} S$ iff $I \operatorname{conf}_{f} S$ and for all instanced functional evolution insfevol $\in \operatorname{InsFEvol}(I) \cap \operatorname{InsFEvol}(S)$ we have that $\exists \bar{t}_{i}, \bar{t}_{s}$ such that

$$
\left(\begin{array}{c}
\text { insfevol } \nabla \bar{t}_{i} \in \operatorname{InsTEvol}(I) \\
\text { insfevol } \nabla \bar{t}_{s} \in \operatorname{InsTEvol}(S) \\
\bar{t}_{i} \leq \bar{t}_{s}
\end{array}\right)
$$

- (weak always) I $\operatorname{conf}_{a}^{w} S$ iff $I \operatorname{conf}_{f} S$ and for all instanced functional evolution insfevol $\in \operatorname{InsFEvol}(I) \cap \operatorname{InsFEvol}(S)$ we have that $\forall \bar{t}_{i}$

$$
\text { insfevol } \nabla \bar{t}_{i} \in \operatorname{InsTEvol}(I) \Longrightarrow \exists \bar{t}_{s}:\left(\begin{array}{c}
\text { insfevol } \nabla \bar{t}_{s} \in \operatorname{InsTEvol}(S) \\
\wedge \\
\sum \bar{t}_{i}=\sum \bar{t}_{s}
\end{array}\right)
$$

- (weak best) $I \operatorname{conf}_{b}^{w} S$ iff $I \operatorname{conf}_{f} S$ and for all instanced functional evolution insfevol $\in \operatorname{InsFEvol}(I) \cap \operatorname{InsFEvol}(S)$ we have that $\forall \bar{t}_{i}$

$$
\text { insfevol } \nabla \bar{t}_{i} \in \operatorname{InsTEvol}(I) \Longrightarrow \forall \bar{t}_{s}:\left(\begin{array}{c}
\text { insfevol } \nabla \bar{t}_{s} \in \operatorname{InsTEvol}(S) \\
\Downarrow \\
\sum \bar{t}_{i} \leq \sum \bar{t}_{s}
\end{array}\right)
$$

- (weak worst) $I \operatorname{conf}_{w}^{w} S$ iff $I \operatorname{conf}_{f} S$ and for all instanced functional evolution insfevol $\in \operatorname{InsFEvol}(I) \cap \operatorname{InsFEvol}(S)$ we have that $\forall \bar{t}_{i}$

$$
\text { insfevol } \nabla \bar{t}_{i} \in \operatorname{InsTEvol}(I) \Longrightarrow \exists \bar{t}_{s}:\left(\begin{array}{c}
\text { insfevol } \nabla \bar{t}_{s} \in \operatorname{InsTEvol}(S) \\
\wedge \\
\sum \bar{t}_{i} \leq \sum \bar{t}_{s}
\end{array}\right)
$$

- (weak sometimes best) $I \operatorname{conf}_{s b}^{w} S$ iff $I \operatorname{conf}_{f} S$ and for all instanced functional evolution insfevol $\in \operatorname{InsFEvol}(I) \cap \operatorname{InsFEvol}(S)$ we have that $\exists \bar{t}_{i}$ such that

$$
\text { insfevol } \nabla \bar{t}_{i} \in \operatorname{InsTEvol}(I) \wedge \forall \bar{t}_{s}:\left(\begin{array}{c}
\text { insfevol } \nabla \bar{t}_{s} \in \operatorname{InsTEvol}(S) \\
\Downarrow \\
\sum \bar{t}_{i} \leq \sum \bar{t}_{s}
\end{array}\right)
$$

- (weak sometimes worst) $I \operatorname{conf}_{s w}^{w} S$ iff $I \operatorname{conf}_{f} S$ and for all instanced functional evolution insfevol $\in \operatorname{InsFEvol}(I) \cap \operatorname{InsFEvol}(S)$ we have that $\exists \bar{t}_{i}, \bar{t}_{s}$ such that

$$
\left(\begin{array}{c}
\text { insfevol } \nabla \bar{t}_{i} \in \operatorname{InsTEvol}(I) \\
\text { insfevol } \nabla \bar{t}_{s} \in \operatorname{InsTEvol}(S) \\
\wedge \\
\sum \bar{t}_{i} \leq \sum \bar{t}_{s}
\end{array}\right)
$$




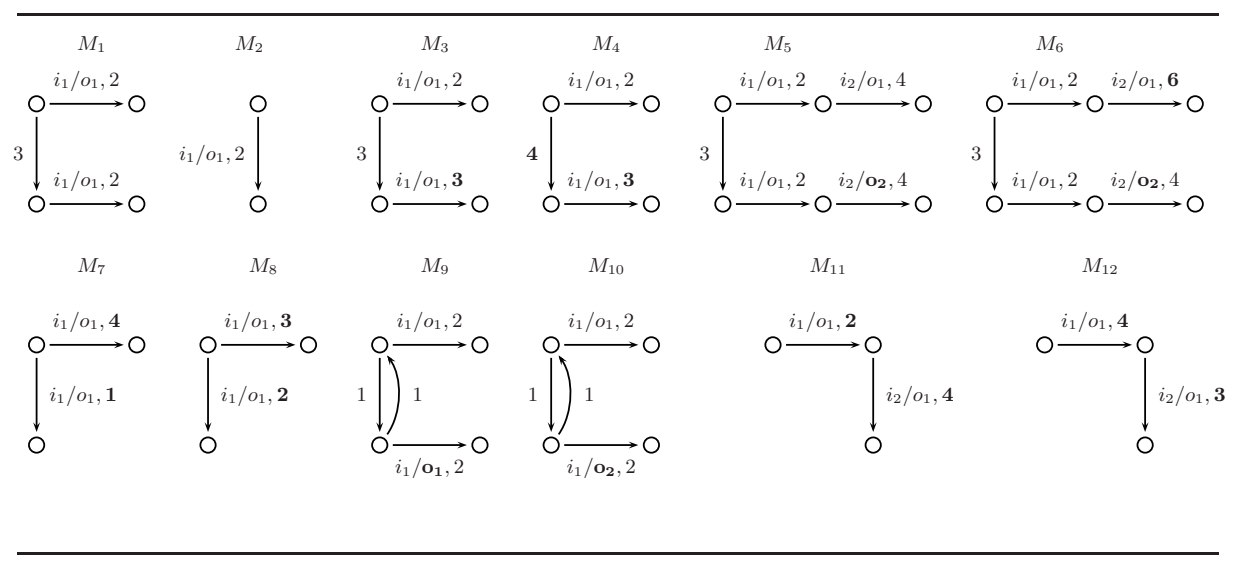

Fig. 3. Example of TEFSMs.

\subsection{Illustrating Examples}

In this section we show how our implementation relations capture the functional and temporal behavior of systems. In particular, we give some examples where several TEFSMs are related. For the sake of simplicity, we will use some additional conformance binary operators. We will assume that $I \operatorname{conf}_{*} S$ denotes that all implementation relations given in Definition 5 hold between $I$ and $S$. If none of these relations holds then we denote it by $I$ cohf $f_{*} S$. Besides, $I$ conf $\square S$ denotes that all relations but $\operatorname{conf}_{a}^{s}$ and conf ${ }_{a}^{w}$ hold. We will consider the TEFSMs depicted in Figure 3. Finally, let us note that if a TEFSM is very similar to the ones presented before, then we stress the differences by using a boldface font.

Equivalent machines. We have $M_{1} \operatorname{conf}_{*} M_{2}$. Actually, we also have $M_{2}$ conf ${ }_{*}$ $M_{1}$. Let us note that the behavior of both machines is exactly the same regardless of whether 3 units of time pass: All transitions available for $M_{1}$ after taking a timeout are also available in $M_{2}$ from its first state. For similar reasons, we have $M_{1} \operatorname{conf}_{*} M_{9}, M_{9} \operatorname{conf}_{*} M_{1}, M_{2} \operatorname{conf}_{*} M_{9}$, and $M_{9} \operatorname{conf}_{*} M_{2}$.

Non-Conformance due to different time values to perform output actions. However, we have $M_{3}$ cohf $_{*} M_{2}$. Let us note that $M_{3}$ may take 3 time units to perform the output $o_{1}$ if it receives the input $i_{1}$ after 3 time units, $\left(3 / i_{1} / 3 / o_{1}\right)$, while $M_{2}$ only needs 2 time units, $\left(3 / i_{1} / 2 / o_{1}\right)$. Moreover, in these machines the only way to perform $i_{1} / o_{1}$ after a timeout 3 consists in taking these traces, respectively (the same applies for traces with a timeout higher than 3 ). Since $M_{3}$ is, in any case, slower than $M_{2}$ for these sequences of inputs/outputs, no conformance relation where $M_{3}$ is the IUT and $M_{2}$ is the specification holds. However, we have $M_{2} \operatorname{conf}_{\square} M_{3}$ : Despite $M_{2}$ does not take the same time values as $M_{3}$ for each sequence, its time is always smaller than (timeouts $\geq 3$ ) or equal to (timeouts $<3$ ) the times of $M_{3}$.

Non-conformance due to different timeouts. As we have seen, reducing the time consumed by actions can benefit a TEFSM with respect to another. In spite of the fact that requirements on timeouts are strict, sometimes having differ- 
ent timeouts can benefit a TEFSM as well. Most traces in $M_{3}$ and $M_{4}$ take the same times. There is an exception: The trace with timeout 3 . In $M_{3}$ we have $\left(3 / i_{1} / 3 / o_{1}\right)$, but in $M_{4}$ we have $\left(3 / i_{1} / 2 / o_{1}\right)$ because after 3 time units pass the state does not change yet in $M_{4}$. Hence, we have $M_{4}$ conf $\square M_{3}$ but $M_{3} \operatorname{cohf} f_{*} M_{4}$.

Non-conformance due to conf $f_{f}$. Let us consider how the availability of outputs affects the relations. We have $M_{5}$ cohf $f_{*} M_{11}$. Let us note that if $i_{2}$ is offered in $M_{11}$ after executing $i_{1} / o_{1}$ then only $o_{1}$ can be produced. However, $M_{5}$ can produce this output as well as $o_{2}$, which is forbidden by $M_{11}$. So, we do not have $M_{5}$ conf ${ }_{f} M_{11}$, and no temporal relation holds without fulfilling this condition. If $M_{5}$ is substituted by $M_{6}$ then the same considerations apply: We have $M_{6}$ cohf $_{*} M_{11}$. However, we have $M_{11}$ conf $\square M_{6}$ because all sequences concerned by $M_{11}$ that appear in $M_{6}$ (in fact only the sequence $i_{1} / o_{1}, i_{2} / o_{1}$ ) are performed faster than or equal to the corresponding trace in $M_{6}$ (but we do not have that all are equal). Let us note that $M_{9} \operatorname{cohf}_{*} M_{10}$ and $M_{10} \operatorname{cohf}_{*} M_{9}$. The reason is that conf $f$ does not hold, though, in this case, it does not hold in either direction. Let us note that, after 1 time units passes and the timeout is raised, if $i_{1}$ is offered then $M_{9}$ must answer $o_{1}$, and $o_{2}$ is forbidden. However, it is the other way around for $M_{10}$. Hence, their answers are mutually incompatible.

Non-conformance due to different time requirements. Let us consider a case where the IUTs and specifications can spent different time values in executing pairs of input/outputs included in traces. We consider $M_{7}$ and $M_{8}$. Since they only perform traces of length 1 , any strong relation coincides with its respective weak version. Next we will refer to strong relations. Both $M_{7}$ and $M_{8}$ can execute $i_{1} / o_{1}$ in a time that cannot be taken in the other, so we do not have $M_{7} \operatorname{conf}_{a}^{s} M_{8}$. The worst time values to execute $i_{1} / o_{1}$ in $M_{7}$ and $M_{8}$ are 4 and 3 , respectively, while the best time values are 1 and 2 , respectively. The worst time of $M_{7}$ is not better than the worst or the best time in $M_{8}$, so we have neither $M_{7} \operatorname{conf}_{w}^{s} M_{8}$ nor $M_{7} \operatorname{conf}_{b}^{s} M_{8}$. However, the best time in $M_{7}$ is better to both the worst and the best time of $M_{8}$. So, both $M_{7} \operatorname{conf}_{s w}^{s} M_{8}$ and $M_{7} \operatorname{conf}_{s b}^{s} M_{8}$ hold. On the other hand, the worst time in $M_{8}$ is better than the worst of $M_{7}$ but not than the best of $M_{7}$. Hence, $M_{8} \operatorname{conf}_{w}^{s} M_{7}$ holds but $M_{8} \operatorname{conf}_{b}^{s} M_{7}$ does not. Finally, the best time in $M_{8}$ is better than the worst of $M_{7}$, but not better than its best one. Thus, $M_{8} \operatorname{conf}_{s w}^{s} M_{7}$ holds, but $M_{8} \operatorname{conf}_{s b}^{s} M_{7}$ does not.

Differences between weak and strong. Next we show how temporal requirements are dealt by strong and weak relations. Let us consider $M_{11}$ and $M_{12}$. No strong relation holds between these TEFSMs in any direction. The reason is that $M_{11}$ performs, $i_{1} / o_{1}$, faster than $M_{12}$, but $M_{12}$ performs the next transition $i_{2} / o_{1}$ faster than $M_{11}$. The result is that none of these machines is always at least as fast as the other (concerning transitions). However, if we consider traces (i.e., weak relations) then some relations arise. Let us note that $M_{11}$ performs both available sequences of inputs/outputs $\left(i_{1} / o_{1}\right.$ and $\left.i_{1} / o_{1}, i_{2} / o_{1}\right)$ faster than $M_{12}$ : In $M_{11}$ they spend 2 and 6 time units, respectively, while these time values are 4 and 7 , respectively, in $M_{12}$. So, all weak relations (but conf ${ }_{a}^{w}$ ) hold: We have $M_{11} \operatorname{conf}_{w}^{w} M_{12}, M_{11} \operatorname{conf}_{b}^{w} M_{12}, M_{11} \operatorname{conf}_{s w}^{w} M_{12}$, and $M_{11} \operatorname{conf}_{s b}^{w} M_{12}$. None of them holds if we exchange the roles of both machines. 


\subsection{Relating Conformance Relations}

Theorem 1. The relations given in Definition 5 are related as follows:

$$
\begin{aligned}
& I \operatorname{conf}_{s w}^{w} S \Leftarrow I \operatorname{conf}_{s b}^{w} S \\
& \Uparrow \quad \Uparrow \\
& I \operatorname{conf}_{a}^{w} S \Rightarrow I \operatorname{conf}_{w}^{w} S \Leftarrow I \operatorname{conf}_{b}^{w} S \\
& \Uparrow \quad \Uparrow \quad \Uparrow \\
& I \operatorname{conf}_{a}^{s} S \Rightarrow I \operatorname{conf}_{w}^{s} S \Leftarrow I \operatorname{conf}_{b}^{s} S \\
& \Downarrow \quad \Downarrow \\
& I \operatorname{conf}_{s w}^{s} S \Leftarrow I \operatorname{conf}_{s b}^{s} S
\end{aligned}
$$

Besides, we have $I \operatorname{conf}_{s w}^{s} S \Rightarrow I \operatorname{conf}_{s w}^{w} S$ and $I \operatorname{conf}_{s b}^{s} S \Rightarrow I \operatorname{conf}_{s b}^{w} S$.

Let us remark that the implications inferred in the previous result are, obviously, transitive. For instance, we also have $I \operatorname{conf}_{a}^{w} S \Rightarrow I \operatorname{conf}_{s w}^{w} S$.

It is interesting to note that if specifications are restricted to take always the same time for each given evolution (independently from the possible derivation taken for such evolution) then, on the one hand, the relations $\operatorname{conf}_{b}^{w}$ and $\operatorname{conf}_{w}^{w}$ would coincide while, on the other hand, $\operatorname{conf}_{b}^{s}$ and $\operatorname{conf}_{w}^{s}$ also coincide. However these relations would be still different from the $\operatorname{conf}_{a}^{w}$ and $\operatorname{conf}_{a}^{s}$ relations. Similarly, if this property holds in implementations then all relations concerning the best temporal traces of the implementation (sometimes relations) coincide with the corresponding relation where all the temporal traces of the implementation are regarded.

Lemma 1. Let us consider two TEFSMs $I=\left(S_{I}, I_{I}, O_{I}, T O_{I}, T r_{I}, s_{i n_{I}}, \bar{y}_{I}\right)$ and $S=\left(S_{S}, I_{S}, O_{S}, T O_{S}, T r_{S}, s_{i n_{S}}, \bar{y}_{S}\right)$. If there do not exist different transitions $\left(s, s^{\prime}, i, o, Q, Z, C\right),\left(s, s^{\prime \prime}, i, o, Q^{\prime}, Z^{\prime}, C^{\prime}\right) \in \operatorname{Tr}_{I}$ then

$$
\begin{aligned}
& I \operatorname{conf}_{b}^{s} S \Leftrightarrow I \operatorname{conf}_{s b}^{s} S \quad I \operatorname{conf}_{w}^{s} S \Leftrightarrow I \operatorname{conf}_{s w}^{s} S \\
& I \operatorname{conf}_{b}^{w} S \Leftrightarrow I \operatorname{conf}_{s b}^{w} S \quad I \operatorname{conf}_{w}^{w} S \Leftrightarrow I \operatorname{conf}_{s w}^{w} S
\end{aligned}
$$

If there do not exist different transitions $\left(s, s^{\prime}, i, o, Q, Z, C\right),\left(s, s^{\prime \prime}, i, o, Q^{\prime}, Z^{\prime}, C^{\prime}\right) \in$ $\operatorname{Tr}_{S}$ then

$$
\begin{array}{ll}
I \operatorname{conf}_{b}^{s} S \Leftrightarrow I \operatorname{conf}_{w}^{s} S & I \operatorname{conf}_{s w}^{s} S \Leftrightarrow I \operatorname{conf}_{s b}^{s} S \\
I \operatorname{conf}_{b}^{w} S \Leftrightarrow I \operatorname{conf}_{w}^{w} S & I \operatorname{conf}_{s w}^{w} S \Leftrightarrow I \operatorname{conf}_{s b}^{w} S
\end{array}
$$

The hierarchy of relations induced in Theorem 1 allows to compare implementations in the following way: $I_{1}$ is preferable to $I_{2}$ to implement $S$ if it meets with $S$ a relation that is stricter according to this hierarchy.

Definition 6. Let $I_{1}, I_{2}$ and $S$ be TEFSMs and $\operatorname{conf}_{x}$ and $\operatorname{conf}_{y}$ be timed conformance relations such that $I_{1} \operatorname{conf}_{x} S, I_{2} \operatorname{conf}_{y} S, \operatorname{conf}_{x} \Rightarrow \operatorname{conf}_{y}$, and $\operatorname{conf}_{y} \nRightarrow \operatorname{conf}_{x}$. We say that $I_{1}$ is preferred to $I_{2}$ to implement $S$ and we denote it by $I_{1}>_{S} I_{2}$. 


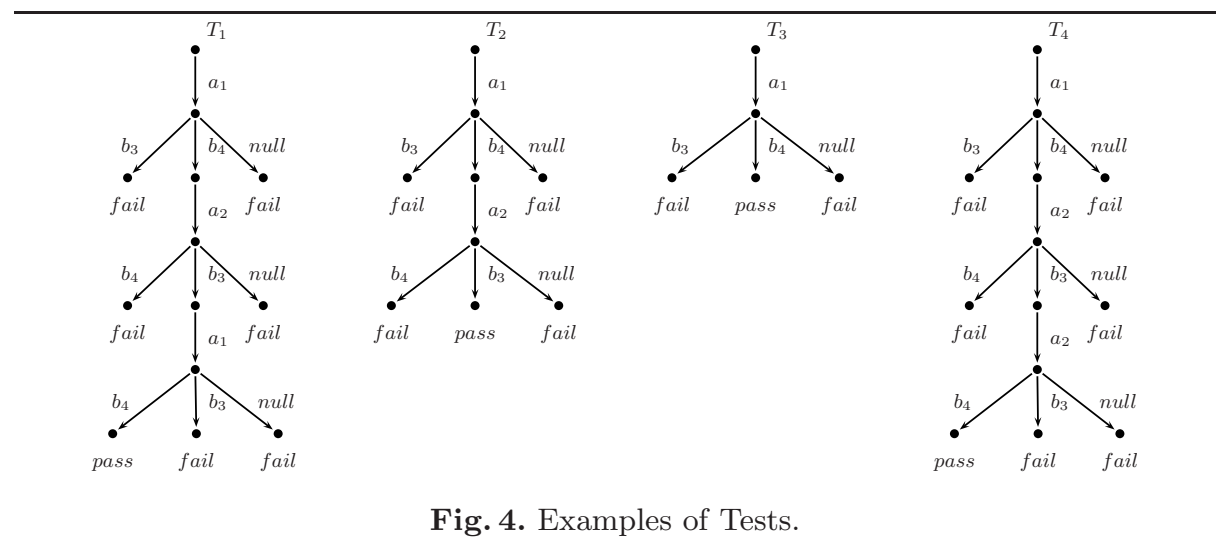

Fig. 4. Examples of Tests.

\section{Definition and Application of Tests}

We consider that tests represent sequences of inputs applied to an IUT. Once an output is received, the tester checks whether it belongs to the set of expected ones or not. In the latter case, a fail signal is produced. In the former case, either a pass signal is emitted (indicating successful termination) or the testing process continues by applying another input. If we are testing an implementation with input and output sets $I$ and $O$, respectively, tests are deterministic acyclic $I / O$ labelled transition systems (i.e. trees) with a strict alternation between an input action and the set of output actions. After an output action we may find either a leaf or another input action. Leaves can be labelled either by pass or by fail. In addition to check the functional behavior of the IUT, test have also to detect whether wrong timed behaviors appear. Thus, tests have to include capabilities to deal with the two ways of specifying time. On the one hand, we will include time stamps to record the time that each sequence of output actions takes to be executed. The time values recorded from the IUT while applying the test will be compared with the ones expected by the specification. Each time stamp will contain a set of time sequences corresponding to the time values that the specification establishes for each transition of a trace. Since we do not restrict non-deterministic behavior, we will have as many time sequences as possible timed evolutions can exist for a trace. Moreover, depending on the number of inputs applied so far, we will have different lengths for the associated time sequences in the time stamps of the test. On the other hand, tests will include delays before offering input actions. The idea is that delays in tests will induce timeouts in IUTs. Thus, we may indirectly check whether the timeouts imposed by the specification are reflected in the IUT by offering input actions after a specific delay. Let us note that a tester can not observe when the IUT takes a timeout. However, she can check the IUT behavior after different delays.

Definition 7. A test is a tuple $T=\left(S, I, O, T r, s_{0}, S_{I}, S_{O}, S_{F}, S_{P}, C, W\right)$ where $S$ is the set of states, $I$ and $O$ are disjoint sets of input and output actions, 
respectively, $\operatorname{Tr} \subseteq S \times(I \cup O) \times S$ is the transition relation, $s_{0} \in S$ is the initial state, and the sets $S_{I}, S_{O}, S_{F}, S_{P} \subseteq S$ are a partition of $S$. The transition relation and the sets of states fulfill the following conditions:

- $S_{I}$ is the set of input states. We have that $s_{0} \in S_{I}$. For all input state $s \in S_{I}$ there exists a unique outgoing transition $\left(s, a, s^{\prime}\right) \in T r$. For this transition we have that $a \in I$ and $s^{\prime} \in S_{O}$.

- $S_{O}$ is the set of output states. For all output state $s \in S_{O}$ we have that for all $o \in O$ there exists a unique state $s^{\prime}$ such that $\left(s, o, s^{\prime}\right) \in \operatorname{Tr}$. In this case, $s^{\prime} \notin S_{O}$. Moreover, there do not exist $i \in I, s^{\prime} \in S$ such that $\left(s, i, s^{\prime}\right) \in T r$.

- $S_{F}$ and $S_{P}$ are the sets of fail and pass states, respectively. We say that these states are terminal. Thus, for all state $s \in S_{F} \cup S_{P}$ we have that there do not exist $a \in I \cup O$ and $s^{\prime} \in S$ such that $\left(s, a, s^{\prime}\right) \in T r$.

Finally, we have two timed functions. $C: S_{P} \longrightarrow \bigcup_{j=1}^{\infty} \mathcal{P}\left(\mathrm{Time}^{j}\right)$ is a function associating time stamps with passing states. $W: S_{I} \longrightarrow$ Time is a function associating delays with input states.

We say that a test $T$ is valid if the graph induced by $T$ is a tree with root at the initial state $s_{0}$.

We say that a test $T$ is an instance of the test $T^{\prime}$ if they only differ in the associated timed functions $C$ and $W$.

Let $\sigma=i_{1} / o_{1}, \ldots, i_{r} / o_{r}$. We write $T \stackrel{\sigma}{\Longrightarrow} s$ if $s \in S_{F} \cup S_{P}$ and there exist states $s_{12}, s_{21}, s_{22}, \ldots s_{r 1}, s_{r 2} \in S$ such that $\left\{\left(s_{0}, i_{1}, s_{12}\right),\left(s_{r 2}, o_{r}, s\right)\right\} \subseteq T r$, for all $2 \leq j \leq r$ we have $\left(s_{j 1}, i_{j}, s_{j 2}\right) \in T r$, and for all $1 \leq j \leq r-1$ we have $\left(s_{j 2}, o_{j}, s_{(j+1) 1}\right) \in T r$.

Let $T$ be a test, $\sigma=i_{1} / o_{1}, \ldots, i_{r} / o_{r}, s^{T}$ be a state of $T$, and $\bar{t}, \overline{t_{o}} \in$ Time $^{r}$. We write $T \stackrel{\sigma}{\Longrightarrow} s^{T}$ if $T \stackrel{\sigma}{\Longrightarrow} s^{T}, t_{1}=D\left(s_{0}\right)$ and for all $1<j \leq r$ we have $t_{j}=D\left(s_{j 1}\right)$.

Let us remark that $T \stackrel{\sigma}{\Longrightarrow} s^{T}$, and its variant $T \stackrel{\sigma}{\Longrightarrow} s^{T}$, imply that $s$ is a terminal state. Next we define the application of a test suite to an implementation. We say that the test suite $\mathcal{T}$ is passed if for all test the terminal states reached by the composition of implementation and test are pass states. Besides, we give different timing conditions in a similar way to what we did for implementation relations.

Definition 8. Let $I$ be a TEFSM, $T$ be a valid test, $\sigma=i_{1} / o_{1}, \ldots, i_{r} / o_{r}, s^{T}$ be a state of $T, \bar{t}=\left(t_{1}, \ldots, t_{r}\right)$, and $\bar{t}_{o}=\left(t_{o 1}, \ldots, t_{o r}\right)$. We write $I \| T \stackrel{\sigma}{\Longrightarrow}_{t} s^{T}$ if $T \stackrel{\sigma}{\Longrightarrow}{ }_{t} s^{T}$ and $(\bar{t}, \sigma) \in \operatorname{InsFEvol}(I)$. We write $I \| T \stackrel{\sigma}{\Longrightarrow} \bar{t}, \bar{t}_{o} s^{T}$ if $I \| T \stackrel{\sigma}{\Longrightarrow}{ }_{t} s^{T}$ and $\left(\bar{t}, \sigma, \bar{t}_{o}\right) \in \operatorname{InsTEvol}(I)$. Let $e=\left(\bar{t}, \sigma, \bar{t}_{o}\right) \in \operatorname{InsTEvol}(I)$. We define the set $\operatorname{Test}(e, \mathcal{T})=\left\{T \mid T \in \mathcal{T} \wedge I \| T \stackrel{\sigma}{\Longrightarrow} \bar{t}_{,} \bar{t}_{o} s^{T}\right\}$.

We say that $I$ passes the set of valid tests $\mathcal{T}$, denoted by $\operatorname{pass}(I, \mathcal{T})$, if for all test $T \in \mathcal{T}$ there do not exist $\sigma, s^{T}, \bar{t}$ such that $I \| T \stackrel{\sigma}{\Longrightarrow}_{\bar{t}} s^{T}$ and $s^{T} \in S_{F}$.

We say that $I$ strongly passes the set of valid tests $\mathcal{T}$ for any time if $\operatorname{pass}(I, \mathcal{T})$ and for all $e=\left(\bar{t}, \sigma, \bar{t}_{o}\right) \in \operatorname{InsTEvol}(I)$ we have that for all $T \in \operatorname{Test}(e, \mathcal{T})$ such that $I \| T \stackrel{\sigma}{\Longrightarrow}{\bar{t}, \bar{t}_{o}}_{s} s^{T}$ it holds $\bar{t}_{o} \in C\left(s^{T}\right)$. 
We say that $I$ strongly passes the set of valid tests $\mathcal{T}$ in the best time if $\operatorname{pass}(I, \mathcal{T})$ and for all $e=\left(\bar{t}, \sigma, \bar{t}_{o}\right) \in \operatorname{InsTEvol}(I)$ we have that for all $T \in$ Test $(e, \mathcal{T})$ such that $I \| T \stackrel{\sigma}{\Longrightarrow} \bar{t}_{,} \bar{t}_{o} s^{T}$, for all $\bar{t}_{c} \in C\left(s^{T}\right)$ it holds $\bar{t}_{o} \leq \bar{t}_{c}$.

We say that $I$ strongly passes the set of valid tests $\mathcal{T}$ in the worst time if $\operatorname{pass}(I, \mathcal{T})$ and for all $e=\left(\bar{t}, \sigma, \bar{t}_{o}\right) \in \operatorname{InsTEvol}(I)$ we have that for all $T \in$ Test $(e, \mathcal{T})$ such that $I \| T \stackrel{\sigma}{\Longrightarrow} \bar{t}_{\bar{t}} \bar{t}_{o} s^{T}$ there exists $\bar{t}_{c} \in C\left(s^{T}\right)$ such that $\bar{t}_{o} \leq \bar{t}_{c}$.

We say that $I$ strongly passes the set of valid tests $\mathcal{T}$ sometimes in best time if $\operatorname{pass}(I, \mathcal{T})$ and there exists $e=\left(\bar{t}, \sigma, \bar{t}_{o}\right) \in \operatorname{InsTEvol}(I)$ such that for all $T \in \operatorname{Test}(e, \mathcal{T})$ with $I \| T \stackrel{\sigma}{\Longrightarrow} \bar{t}_{, \bar{t}_{o}} s^{T}$ we have that for all $\bar{t}_{c} \in C\left(s^{T}\right)$ it holds $\bar{t}_{o} \leq \bar{t}_{c}$.

We say that $I$ strongly passes the set of valid tests $\mathcal{T}$ sometimes in worst time if $\operatorname{pass}(I, \mathcal{T})$ and there exists $e=\left(\bar{t}, \sigma, \bar{t}_{o}\right) \in \operatorname{InsTEvol}(I)$ such that for all $T \in \operatorname{Test}(e, \mathcal{T})$ with $I \| T \stackrel{\sigma}{\Longrightarrow} \bar{t}_{,} \bar{t}_{o} s^{T}$ we have that there exists $\bar{t}_{c} \in C\left(s^{T}\right)$ such that $\bar{t}_{o} \leq \bar{t}_{c}$.

We say that $I$ weakly passes the set of valid tests $\mathcal{T}$ for any time if pass $(I, \mathcal{T})$ and for all $e=\left(\bar{t}, \sigma, \bar{t}_{o}\right) \in \operatorname{InsTEvol}(I)$ we have that for all $T \in \operatorname{Test}(e, \mathcal{T})$ such that $I \| T \stackrel{\sigma}{\Longrightarrow} \bar{t}_{, \bar{t}_{o}} s^{T}$ it holds $\sum \bar{t}_{o}=\sum \bar{t}_{c}$ for some $\bar{t}_{c} \in C\left(s^{T}\right)$.

We say that $I$ weakly passes the set of valid tests $\mathcal{T}$ in the best time if $\operatorname{pass}(I, \mathcal{T})$ and for all $e=\left(\bar{t}, \sigma, \bar{t}_{o}\right) \in \operatorname{InsTEvol}(I)$ we have that for all $T \in$ Test $(e, \mathcal{T})$ such that $I \| T \stackrel{\sigma}{\Longrightarrow} \bar{t}_{, \bar{t}_{o}} s^{T}$, for all $\bar{t}_{c} \in C\left(s^{T}\right)$ it holds $\sum \bar{t}_{o} \leq \sum \bar{t}_{c}$.

We say that $I$ weakly passes the set of valid tests $\mathcal{T}$ in the worst time if $\operatorname{pass}(I, \mathcal{T})$ and for all $e=\left(\bar{t}, \sigma, \bar{t}_{o}\right) \in \operatorname{InsTEvol}(I)$ we have that for all $T \in$ Test $(e, \mathcal{T})$ such that $I \| T \stackrel{\sigma}{\Longrightarrow} \bar{t}_{,} \bar{t}_{o} s^{T}$ there exists $\bar{t}_{c} \in C\left(s^{T}\right)$ such that $\sum \bar{t}_{o} \leq$ $\sum \bar{t}_{c}$.

We say that $I$ weakly passes the set of valid tests $\mathcal{T}$ sometimes in best time if $\operatorname{pass}(I, \mathcal{T})$ and there exists $e=\left(\bar{t}, \sigma, \bar{t}_{o}\right) \in \operatorname{InsTEvol}(I)$ such that for all $T \in \operatorname{Test}(e, \mathcal{T})$ with $I \| T \stackrel{\sigma}{\Longrightarrow} \bar{t}_{, \bar{t}_{o}} s^{T}$ we have that for all $\bar{t}_{c} \in C\left(s^{T}\right)$ it holds $\sum \bar{t}_{o} \leq \sum \bar{t}_{c}$.

We say that $I$ weakly passes the set of valid tests $\mathcal{T}$ sometimes in worst time if $\operatorname{pass}(I, \mathcal{T})$ and there exists $e=\left(\bar{t}, \sigma, \bar{t}_{o}\right) \in \operatorname{InsTEvol}(I)$ such that and for all $T \in \operatorname{Test}(e, \mathcal{T})$ with $I \| T \stackrel{\sigma}{\Longrightarrow} \bar{t}_{,} \bar{t}_{o} s^{T}$ we have that there exists $\bar{t}_{c} \in C\left(s^{T}\right)$ such that $\sum \bar{t}_{o} \leq \sum \bar{t}_{c}$.

\section{Conclusions and Future Work}

In this paper we have introduced a new model to specify timed systems. In contrast with most approaches, our formalism allows to define time in two different ways: Duration of actions and timeouts of the system. Thus, by separating these two notions, it is easier to specify temporal properties of systems than if we use a formalism where only one of the possibilities is available. We have also developed a testing theory. On the one hand, we have defined ten conformance relations that take into account the influence of non-determinism in the behavior of systems. On the other hand, we have introduced a notion of test. In order to 
capture the timed behavior of the IUT, test can both delay the execution of the IUT and record the time that it took to perform a given action.

In terms of future work, we would like to take this paper as a first step, together with [11], to define a testing theory for systems presenting stochastic time together with timeouts.

\section{References}

1. R. Alur and D. Dill. A theory of timed automata. Theoretical Computer Science, 126:183-235, 1994

2. B.S. Bosik and M.U. Uyar. Finite state machine based formal methods in protocol conformance testing. Computer Networks \& ISDN Systems, 22:7-33, 1991.

3. E. Brinksma and J. Tretmans. Testing transition systems: An annotated bibliography. In 4th Summer School, MOVEP 2000, LNCS 2067, pages 187-195. Springer, 2001.

4. D. Clarke and I. Lee. Automatic generation of tests for timing constraints from requirements. In 3rd Workshop on Object-Oriented Real-Time Dependable Systems, 1997.

5. K. El-Fakih, N. Yevtushenko, and G. von Bochmann. FSM-based incremental conformance testing methods. IEEE Transactions on Software Engineering, 30(7):425436, 2004.

6. A. En-Nouaary and R. Dssouli. A guided method for testing timed input output automata. In TestCom 2003, LNCS 2644, pages 211-225. Springer, 2003.

7. M.A. Fecko, M.Ü. Uyar, A.Y. Duale, and P.D. Amer. A technique to generate feasible tests for communications systems with multiple timers. IEEE/ACM Transactions on Networking, 11(5):796-809, 2003.

8. T. Higashino, A. Nakata, K. Taniguchi, and A. Cavalli. Generating test cases for a timed I/O automaton model. In 12th Workshop on Testing of Communicating Systems, pages 197-214. Kluwer Academic Publishers, 1999.

9. D. Lee and M. Yannakakis. Principles and methods of testing finite state machines: A survey. Proceedings of the IEEE, 84(8):1090-1123, 1996.

10. M. Núñez and I. Rodríguez. Encoding PAMR into (timed) EFSMs. In FORTE 2002, LNCS 2529, pages 1-16. Springer, 2002.

11. M. Núñez and I. Rodríguez. Towards testing stochastic timed systems. In FORTE 2003, LNCS 2767, pages 335-350. Springer, 2003.

12. M. Núñez and I. Rodríguez. Conformance testing relations for timed systems. In 5th Int. Workshop on Formal Approaches to Software Testing (FATES 2005), LNCS 3997, pages 103-117. Springer, 2006.

13. J.C. Park and R.E. Miller. Synthesizing protocol specifications from service specifications in timed extended finite state machines. In 17th IEEE Int. Conf. on Distributed Computing Systems, ICDCS'97, pages 253-260. IEEE Computer Society, 1997.

14. A. Petrenko. Fault model-driven test derivation from finite state models: Annotated bibliography. In 4th Summer School, MOVEP 2000, LNCS 2067, pages 196-205. Springer, 2001.

15. J. Springintveld, F. Vaandrager, and P.R. D'Argenio. Testing timed automata. Theoretical Computer Science, 254(1-2):225-257, 2001.

16. J. Tretmans. Test generation with inputs, outputs and repetitive quiescence. Software - Concepts and Tools, 17(3):103-120, 1996. 